\title{
Adaptações Fisiológicas Precoces e Tardias após Valvotomia Mitral por Cateter Balão
}

\author{
Japy Angelini O liveira Fo, Marcelo Regazzini, O rlando Campos Fo, A na Fátima Salles, Turíbio Leite \\ Barros N eto, N eil Ferreira Novo, José Bocanegra, Eulógio Martinez Foo, Dirceu Vieira Santos Fo
}

São Paulo, SP

Objetivo - Avaliação não invasiva das respostas cardiopulmonares ao exercício a curto e longo prazo após valvotomia mitral por cateter balão.

Métodos - Estudaram-se 9 pacientes do sexo feminino, $35 \pm 9$ anos, com estenose mitral, tipo funcional II ou III, em uso de digitálicos e diuréticos, através de teste ergoespirométrico, eletrocardiograma e ecocardiograma, antes e após valvotomia em fase precoce ( 3 a 5 dias) e tardia (8 a 12 meses).

Resultados - Os pacientes evoluíram para tipo funcional II (44\%) ou I (56\%), na fase tardia. Houve redução da freqüência cardíaca de repouso $(87 \pm 11 \mathrm{bpm} v \mathrm{~s}$ $85 \pm 7 \mathrm{bpm}$ vs $75 \pm 9 \mathrm{bpm}$ ) e elevação do número de estágios (4 \pm 1 estágios vs $5 \pm 2$ estágios vs $6 \pm 1$ estágios); a capacidade aeróbia máxima aumentou apenas na fase tardia $(16 \pm 3 \mathrm{~mL} / \mathrm{kg} / \mathrm{min} v \mathrm{~s} 18 \pm 4 \mathrm{~mL} / \mathrm{kg} / \mathrm{min}$ vs $22 \pm 7 \mathrm{~mL} / \mathrm{kg} / \mathrm{min})$. $O$ limiar anaeróbio, a ventilação pulmonar e o equivalente ventilatório do $\mathrm{O}_{2}$ permaneceram inalterados. Nas cargas submáximas de exercício ocorreu redução da freqüência cardíaca (estágio I: $124 \pm 18 \mathrm{bpm}$ vs $112 \pm 13 \mathrm{bpm}$ vs $87 \pm 15 \mathrm{bpm}$ ), consumo de $\mathrm{O}_{2}$ (estágio I: $10 \pm 2 \mathrm{~mL} / \mathrm{kg} / \mathrm{min} v \mathrm{~s}$ $8 \pm 2 \mathrm{~mL} / \mathrm{kg} / \mathrm{min}$ vs $8 \pm \mathrm{mL} / \mathrm{kg} / \mathrm{min}$ ) e ventilação pulmonar, nas fases precoce e tardia. A área valvar mitral mostrou reduções na fase tardia $\left(0,94 \mathrm{~cm}^{2}\right.$ vs $1,66 \mathrm{~cm}^{2}$ vs $\left.1,20 \mathrm{~cm}^{2}\right)$.

Conclusão - Apesar da tendência à reestenose parcial, houve melhora no tipo funcional e no desempenho cardiopulmonar com diminuição da sobrecarga circulatória no exercício submáximo.

Palavras-chave: estenose mitral, valvoplastia mitral, teste ergométrico, ecocardiograma

\section{Early and Late Physiological Effects of Balloon Mitral Valvuloplasty}

Purpose - To evaluate the early and late cardiorespiratory responses after balloon mitral valvuloplasty.

Methods - Nine female patients aged $35 \pm 9$ years, with mitral stenosis, in class II or III (NYHA) underwent upright ergoespirometric test, resting electrocardiogram and echocardiogram before, 3 to 5 days (early evaluation) and 8 to 12 months (late evaluation) after mitral valvuloplasty. All patients were treated with digitalis and diuretics.

Results - During late evaluation, $44 \%$ patients were in class II and $56 \%$ were in class I (NYHA). The resting heart rate decreased $(87 \pm 11 \mathrm{bpm} v \mathrm{~s} 85 \pm 7 \mathrm{bpm} v \mathrm{v} 75 \pm 9 \mathrm{bpm})$ and the number of steps increased ( $4 \pm 1$ steps vs $5 \pm 2$ steps vs $6 \pm 1$ steps); the peak oxygen uptake improved only in the late evaluation $(16 \pm 3 \mathrm{~mL} / \mathrm{kg} / \mathrm{min}$ vs $18 \pm 4 \mathrm{~mL} / \mathrm{kg} / \mathrm{min}$ vs $22 \pm 7 \mathrm{~mL} / \mathrm{kg} / \mathrm{min}$ ). The anaerobic threshold, minute ventilation (VE) and ventilatory equivalent for oxygen showed no change. The heart rate ( $1^{\text {st }}$ step: $124 \pm 18 \mathrm{bpm} v \mathrm{~s}$ $112 \pm 13 \mathrm{bpm}$ vs $87 \pm 15 \mathrm{bpm}$ ), $\mathrm{O}_{2}$ uptake ( ${ }^{\text {st }}$ step: $10 \pm 2 \mathrm{~mL} /$ $\mathrm{kg} / \mathrm{min}$ vs $8 \pm 2 \mathrm{~mL} / \mathrm{kg} / \mathrm{min}$ vs $8 \pm 2 \mathrm{~mL} / \mathrm{kg} / \mathrm{min}$ ) and $V E \mathrm{de}$ creased during submaximal exercise in early and late phases. The mitral valve area decreased in the late evaluation $\left(0.94 \mathrm{~cm}^{2}\right.$ vs $1.66 \mathrm{~cm}^{2}$ vs $\left.1.20 \mathrm{~cm}^{2}\right)$.

Conclusion - Although partial restenosis tended to occur in these patients, they improved the functional class and cardiorespiratory performance and cardiocirculatory load during submaximal exercise.

Key-words: mitral stenosis, mitral valvuloplasty, exercise test, echocardiogram

Arq Bras Cardiol, volume 70 (n' 2), 81-86, 1998

Escola Paulista de Medicina - UNIFESP

Correspondência: Japy Angelini Oliveira F $F^{\circ}$ Rua Tapejara, 109 - 05594-050 - São Paulo, SP

Recebido para publicação em 1/9/97

Aceito em 25/11/97
A valvotomia mitral por cateter balão(VTM) é um tratamento eficaz e alternativo à cirurgia da valva mitral. O alívio dos sintomas, o aumento da duração do exercício e a melhora das variáveis hemodinâmicas e cardiorrespiratórias têm sido descritos nos primeiros meses após valvotomia ${ }^{1-7}$. 
Entretanto, elevações no pico de consumo de $\mathrm{O}_{2}$ e limiar anaeróbio não foram verificadas em testes realizados logo após a VTM ${ }^{8}$. Alterações periféricas musculares têm sido relatadas três meses após VTM, melhorando a capacidade aeróbia durante o exercício ${ }^{9}$.

O presente artigo descreve a evolução e curto e longo prazo das variáveis metabólicas cardiorrespiratórias de amostra de pacientes submetidos à VTM ${ }^{10}$.

\section{Métodos}

Submeteram-se à VTM por cateter-balão nove portadores de estenose valvar mitral predominante, em tipo funcional (TF) II e III (NYHA). Os pacientes eram do sexo feminino com idades de $35 \pm 9$ anos e faziam uso de digitálicos, diuréticos e cloreto de potássio. Todos os pacientes foram esclarecidos sobre objetivos e riscos do estudo, tendo concordado de livre e espontânea vontade em participar da pesquisa.

Foram realizados exame clínico, eletrocardiograma (ECG), ecodopplercardiograma, teste ergoespirométrico (TEE), antes e após VPM em intervalo de tempo precoce de três a cinco dias e tardio de oito a 12 meses. Ecocardiogramas foram feitos em aparelho ultra-sônico ATL-MK (Seattle, USA) com transdutor de 3,0MHz. A área da valva mitral foi obtida através do tempo meia-pressão ${ }^{11}$. Os TEE foram sintomas limitantes, realizados em esteira rolante na

\begin{tabular}{|ccccccc|}
\hline \multicolumn{5}{|c|}{ Tabela I - Protocolo de teste ergoespirométrico em esteira } \\
\hline $\begin{array}{c}\text { Estágio } \\
\left(\mathrm{n}^{\circ}\right)\end{array}$ & $\begin{array}{c}\text { Velocidade } \\
(\mathrm{MPH})\end{array}$ & $\begin{array}{c}\text { Inclinação } \\
(\%)\end{array}$ & $\begin{array}{c}\text { Duração } \\
(\mathrm{min})\end{array}$ & $\begin{array}{c}\mathrm{VO}_{2} \\
\mathrm{mLO}_{2} / \mathrm{kg} / \mathrm{min}\end{array}$ & $\begin{array}{c}\text { Predito* } \\
\text { MET }\end{array}$ & $\begin{array}{c}\text { Predito* } \\
\text { M }\end{array}$ \\
\hline 1 & 1,0 & 0 & 3 & 8 & 2,3 \\
2 & 2,0 & 2 & 3 & 14 & 4,0 \\
3 & 2,0 & 4 & 3 & 16 & 4,6 \\
4 & 2,0 & 6 & 3 & 18 & 5,1 \\
5 & 2,5 & 6 & 3 & 20 & 5,7 \\
6 & 3,0 & 6 & 3 & 24 & 6,9 \\
7 & 3,0 & 8 & 3 & 27 & 7,7 \\
\hline \multirow{7}{*}{ *omograma de Shephard }
\end{tabular}

vigência de medicação usual, através de protocolo apropriado às condições dos pacientes e com incremento de carga aproximado de $1 \mathrm{MET}$ em cada estágio (tab. I). A aferição da pressão arterial (PA) foi realizada através de esfigmomanômetro de coluna de mercúrio, pelo mesmo experimentador durante o TEE. A frequiência cardíaca (FC) foi aferida através da média de 10 intervalos RR consecutivos no ECG. A análise das trocas gasosas foi realizada através de gasômetro Tissot (Collins, USA) de capacidade de 120L, analisador de $\mathrm{CO}_{2}$ Beckman, modelo LB-2 (USA) e analisador de $\mathrm{O}_{2}$ Beckman, modelo OM-11 (USA). Utilizou-se máscara respiratória de baixa resistência (Vacumed, USA). O consumo de oxigênio $\left(\mathrm{VO}_{2}\right)$ no limiar anaeróbio (LA) foi definido como o $\mathrm{VO}_{2}$ no qual o equivalente ventilatório do

\begin{tabular}{|c|c|c|c|c|c|c|}
\hline \multirow[t]{2}{*}{ Paciente } & \multicolumn{3}{|c|}{ Tipo funcional } & \multicolumn{3}{|c|}{ Área valvar mitral } \\
\hline & pré-VTM & pós-I-VTM & pós-II-VTM & pré-VTM & pós-I- VTM & pós-II- VTM \\
\hline 1 & II & II & II & 1,40 & 2,70 & 1,80 \\
\hline 2 & II & II & II & 1,30 & 1,40 & 1,30 \\
\hline 4 & II & II & II & 0,90 & 1,50 & 0,98 \\
\hline 5 & III & II & II & 0,80 & 1,40 & 0,98 \\
\hline 6 & III & II & I & 0,70 & 1,00 & 0,81 \\
\hline 7 & III & III & I & 0,90 & 1,40 & 1,25 \\
\hline 8 & III & I & I & 0,90 & 1,30 & 1,30 \\
\hline 9 & III & I & I & 0,90 & 1,80 & 1,30 \\
\hline
\end{tabular}

\begin{tabular}{|c|c|c|c|c|c|c|}
\hline & $\begin{array}{c}\mathrm{VO}_{2} \text { pico } \\
(\mathrm{mL} / \mathrm{kg} / \mathrm{min})\end{array}$ & $\begin{array}{c}\mathrm{LA} \\
(\mathrm{mL} / \mathrm{kg} / \mathrm{min})\end{array}$ & $\begin{array}{l}\mathrm{LA} \% \\
(\%)\end{array}$ & $\begin{array}{c}\text { VE BTPS } \\
\text { (L/min BTPS) }\end{array}$ & $\begin{array}{c}\mathrm{VEO}_{2} \\
(1 / \mathrm{min} \mathrm{BTPS})\end{array}$ & $\begin{array}{c}\mathrm{VEO}_{2} \\
(\mathrm{~L} / \mathrm{min} \mathrm{BTPS})\end{array}$ \\
\hline Pré-VTM & $16 \pm 3$ & $13 \pm 2$ & $77 \pm 8$ & $36 \pm 9$ & $42 \pm 4$ & $36 \pm 5$ \\
\hline Pós I-VTM & $18 \pm 4$ & $14 \pm 3$ & $75 \pm 9$ & $37 \pm 10$ & $39 \pm 5$ & $34 \pm 4$ \\
\hline Pós II-VTM & $22 \pm 7$ & $15 \pm 3$ & $73 \pm 16$ & $40 \pm 9$ & $40 \pm 6$ & $39 \pm 3$ \\
\hline \multicolumn{7}{|c|}{ Teste de Tukey } \\
\hline $1 \times 2$ & 2,00 & $3 / 4$ & $3 / 4$ & $3 / 4$ & $3 / 4$ & 2,44 \\
\hline $1 \times 3$ & $5,56^{*}$ & $3 / 4$ & $3 / 4$ & $3 / 4$ & $3 / 4$ & 3,22 \\
\hline $2 \times 3$ & 3,56 & $3 / 4$ & $3 / 4$ & $3 / 4$ & $3 / 4$ & $5,67^{*}$ \\
\hline
\end{tabular}


$\mathrm{O}_{2}\left(\mathrm{VEO}_{2}\right)$ era mínimo, seguindo-se de aumento progressivo; o $\mathrm{VO}_{2}$ após o qual ocorreu um aumento não linear da ventilação pulmonar (VE) e o $\mathrm{VO}_{2}$ onde a razão das trocas respiratórias aumentou mais intensamente. VE foi aferida em BTPS (condição de temperatura corporal, pressão atmosférica ambiental com vapor de água totalmente saturado).

Na análise estatística utilizaram-se: análise de variância para grupos não independentes, complementada pelo teste de Tukey; análise de variância por postos de Friedman, complementada pelo teste de comparações múltiplas; e o teste de concordância de Kappa. Em todos os testes fixouse em 0,05 ou $5 \%$ (p£0,05) o nível para rejeição da hipótese de nulidade, assinalando-se com um asterisco $(*)$ os valores estatisticamente significantes.

\section{Resultados}

Os resultados referentes às avaliações pré-valvotomia mitral (pré-VTM) três a cinco dias pós-valvotomia mitral (pós-I-VTM) e oito a 12 meses pós-valvotomia mitral (pósII-VTM) estão resumidos nas tabelas II a V.

A tabela II traz a evolução do TF (NYHA). Nenhum dos pacientes apresentou sintomas de dor anginosa durante a evolução. Embora tenha-se constatado tendência para a melhora do TF, a variação não foi significativa, provavelmente, dado o pequeno número da amostra. Houve redução significante da FC de repouso nos valores pós II VTM (87 $\pm 11 \mathrm{bpm}, 85 \pm 7 \mathrm{bpm}, 75 \pm 9 \mathrm{bpm} *$ ). As médias referentes a PA sistólica (PAS) de repouso $(109 \pm 1 \mathrm{mmHg}, 109 \pm 10 \mathrm{mmHg}$, $112 \pm 14 \mathrm{mmHg}$ ) e a PA diastólica (PAD) de repouso (71 \pm $6 \mathrm{mmHg}, 68 \pm 3 \mathrm{mmHg}, 71 \pm 8 \mathrm{mmHg}$ ), respectivamente, pré, pós I e pós II VTM, não mostraram alterações significativas. Não se registraram evidências clínicas de reestenose e ou insuficiência mitral.

Os valores referentes à média da área valvar mitral foram de, respectivamente, $0,94 \mathrm{~cm}^{2}, 1,66 \mathrm{~cm}^{2} *$ e $1,20 \mathrm{~cm}^{2}$ nas fases pré, pós-I e pós-II-VTM. As medidas referentes aos pacientes nas três fases foram submetidas a análise de variância para os postos de Friedman e complementadas pelo teste de comparações múltiplas. As médias da fase pós-I foram significativamente mais elevadas que os respectivos valores das fases pré e pós-II (tab. II).
Sete pacientes permaneceram em ritmo sinusal e dois pacientes em fibrilação atrial durante a evolução. Padrões de sobrecarga ventricular direita e sobrecarga atrial esquerda mostraram tendência não significativa à regressão. $\mathrm{O}$ eixo elétrico do coração diminuiu significativamente, desviando-se para a esquerda: $86 \pm 17$ graus, $51 \pm 37 *$ graus, $56 \pm 40 *$ graus, respectivamente, nos valores pré, pós-I e pós-II-VTM.

Os TEE foram realizados sem acidentes e o exercício foi interrompido em todos os casos por exaustão. Desníveis anormais de segmento ST ocorreram em, respectivamente, cinco casos, dois casos nas fases pré, pós-I e pós-II-VTM; a média dos desníveis máximos ocorridos foi semelhante nas três fases: $0,1 \mathrm{mV} ; 0,1 \mathrm{mV} ; 0,1 \mathrm{mV}$.

Observou-se aumento significante na duração dos TEE (pré: $4 \pm 1$ números totais de estágio vs pós-I: $5 \pm 2$ números totais de estágios* vs pós-II: $6 \pm 1$ números totais de estágios*). Não houve variações significantes com relação a

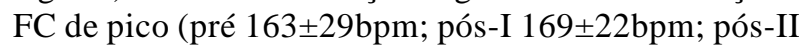
$159 \pm 22 b p m$ ), PAS de pico (pré $112 \pm 26 \mathrm{mmHg}$; pós-I $123 \pm 17 \mathrm{mmHg}$; pós-II $117 \pm 23 \mathrm{mmHg}$ ) e das PAD (pré

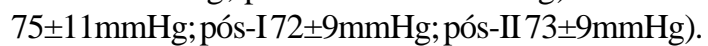

Nas tabelas III, IV, V e VI são apresentados os resultados relativos às variáveis metabólicas. Não houve diferenças significantes entre as médias de $\mathrm{VO}_{2}$ pico, pré e pós-IVTM; entretanto, na evolução tardia, registrou-se elevação significativa (pré $16 \pm 3 \mathrm{mLO}_{2} \mathrm{~kg} / \mathrm{min}$; pós-I $18 \pm 4 \mathrm{mLO}_{2} \mathrm{~kg} / \mathrm{min}$, pós-II $22 \pm 7 \mathrm{mLO}_{2} \mathrm{~kg} / \mathrm{min}^{*}$ ).Não ocorreram variações significantes no limiar anaeróbio, em valores absolutos e relativos, e nos valores de pico da $\mathrm{VEe}$ do $\mathrm{VEO}_{2}$. Entretanto, registraram-se elevações significativas tardias no pico do equivalente ventilatório para $\mathrm{CO}_{2}$ (pré $36 \pm 5 \mathrm{~L} / \mathrm{min}$; pós-I

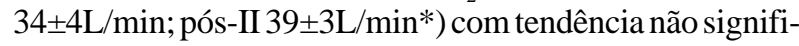
cativa de elevação tardia dos valores de pico da VE.

Observaram-se reduções significativas de FC no $1^{\circ}$ estágio (pré: $124 \pm 18 \mathrm{bpm}$; pós-I $112 \pm 13 \mathrm{bpm} *$; pós-II $87 \pm 15$ bpm*); no $2^{\circ}$ estágio (pré $144 \pm 22$ bpm; pós-I $121 \pm 12 b^{b}{ }^{*}$; pós-II $108 \pm 12 b p m *$ ), no $3^{\circ}$ estágio (pré

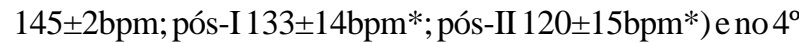
estágio pré $162 \pm 21 \mathrm{bpm}$; pós-I $148 \pm 11 \mathrm{bpm} *$; pós-II $127 \pm 16 \mathrm{bpm} *$ ).

Após VTM, o $\mathrm{VO}_{2}$ mostrou reduções significantes no

\begin{tabular}{|c|c|c|c|c|c|}
\hline \multicolumn{6}{|c|}{ Tabela IV - Variáveis circulatórias e metabólicas no $1^{\circ}$ estágio do teste ergoespirométrico de pacientes pré e pós valvotomia mitral } \\
\hline & $\begin{array}{c}\text { FC } \\
(\mathrm{bpm})\end{array}$ & $\begin{array}{c}\mathrm{VO}_{2} \\
\mathrm{~mL} / \mathrm{kg} / \mathrm{min}\end{array}$ & $\begin{array}{c}\text { VE BTPS } \\
\text { L/min BTPS }\end{array}$ & $\begin{array}{c}\mathrm{VEO}_{2} \\
\mathrm{~L} / \mathrm{min} \text { BTPS }\end{array}$ & $\begin{array}{c}\text { VECO2 } \\
\text { 1/min BTPS }\end{array}$ \\
\hline Pré-VTM & $124 \pm 18$ & $10 \pm 2$ & $22 \pm 6$ & $42 \pm 6$ & $40 \pm 7$ \\
\hline Pós-I-VTM & $112 \pm 13$ & $8 \pm 2$ & $15 \pm 5$ & $41 \pm 6$ & $37 \pm 5$ \\
\hline Pós-II-VTM & $87 \pm 15$ & $8 \pm 2$ & $15 \pm 3$ & $38 \pm 6$ & $45 \pm 7$ \\
\hline \multicolumn{6}{|c|}{ Teste de Tukey } \\
\hline $1 \times 2$ & 15,33 & $2,78 *$ & $7,00 *$ & $3 / 4$ & $3 / 4$ \\
\hline $1 \times 3$ & $40,11 *$ & 2,67 & $7,22 *$ & $3 / 4$ & $3 / 4$ \\
\hline \multicolumn{6}{|l|}{$2 \times 3$} \\
\hline & 0,11 & 0,22 & $3 / 4$ & $3 / 4$ & \\
\hline
\end{tabular}




\begin{tabular}{|c|c|c|c|c|c|}
\hline & $\begin{array}{c}\mathrm{FC} \\
(\mathrm{bpm})\end{array}$ & $\begin{array}{c}\mathrm{VO}_{2} \\
\mathrm{~mL} / \mathrm{kg} / \mathrm{min}\end{array}$ & $\begin{array}{c}\text { VE BTPS } \\
\text { L/min BTPS }\end{array}$ & $\begin{array}{c}\mathrm{VEO}_{2} \\
\mathrm{~L} / \mathrm{min} \mathrm{BTPS}\end{array}$ & $\begin{array}{c}\mathrm{VECO}_{2} \\
\mathrm{~L} / \mathrm{min} \mathrm{BTPS}^{2}\end{array}$ \\
\hline Pré-VTM & $144 \pm 22$ & $13 \pm 3$ & $25 \pm 4$ & $40 \pm 4$ & $36 \pm 4$ \\
\hline Pós I-VTM & $121 \pm 12$ & $10 \pm 2$ & $18 \pm 3$ & $34 \pm 3$ & $34 \pm 3$ \\
\hline Pós-II-VTM & $108 \pm 12$ & $11 \pm 2$ & $21 \pm 4$ & $35 \pm 12$ & $40 \pm 13$ \\
\hline \multicolumn{6}{|l|}{ Teste de Tukey } \\
\hline $1 \times 2$ & $22,56^{*}$ & $3,00 *$ & $8,11 *$ & $5,11 *$ & 1,56 \\
\hline $1 \times 3$ & $36,22 *$ & $2,11^{*}$ & $4,56^{*}$ & $5,33^{*}$ & $4,11 *$ \\
\hline $2 \times 3$ & 13,67 & 0,89 & $3,56^{*}$ & 0,22 & $5,67 *$ \\
\hline
\end{tabular}

\begin{tabular}{|c|c|c|c|c|c|}
\hline \multicolumn{6}{|c|}{ Tabela VI - Variáveis circulatórias e metabólicas no $3^{\circ}$ estágio do teste ergoespirométrico de pacientes pré e pós valvotomia mitral } \\
\hline & $\begin{array}{c}\mathrm{FC} \\
(\mathrm{bpm})\end{array}$ & $\begin{array}{c}\mathrm{VO}_{2} \\
\mathrm{~mL} / \mathrm{kg} / \mathrm{min}\end{array}$ & $\begin{array}{c}\text { VE BTPS } \\
\text { L/min BTPS }\end{array}$ & $\begin{array}{c}\mathrm{VEO}_{2} \\
\mathrm{~L} / \mathrm{min} \mathrm{BTPS}\end{array}$ & $\begin{array}{c}\mathrm{VECO}_{2} \\
\mathrm{~L} / \mathrm{min} \mathrm{BTPS}\end{array}$ \\
\hline Pré-VTM & $145 \pm 21$ & $14 \pm 2$ & $28 \pm 3$ & $40 \pm 3$ & $34 \pm 4$ \\
\hline Pós I-VTM & $133 \pm 14$ & $3 \pm 2$ & $21 \pm 4$ & $34 \pm 4$ & $33 \pm 4$ \\
\hline Pós II-VTM & $120 \pm 15$ & $12 \pm 2$ & $23 \pm 5$ & $35 \pm 12$ & $38 \pm 12$ \\
\hline \multicolumn{6}{|l|}{ Teste de Tukey } \\
\hline $1 \times 2$ & $10,86^{*}$ & $3 / 4$ & $6,75^{*}$ & $5,13^{*}$ & 1,56 \\
\hline $1 \times 3$ & $25,13^{*}$ & $3 / 4$ & $5,13 *$ & $3,88 *$ & $3,38 *$ \\
\hline $2 \times 3$ & $14,25^{*}$ & $3 / 4$ & 1,63 & 1,25 & $4,75^{*}$ \\
\hline
\end{tabular}

$1^{\circ}$ e $2^{\circ}$ estágios, como mostram as tabelas IV e V. A análise da VE revelou alterações significativas nos $1^{\circ}, 2^{\circ}$ e $3^{\circ}$ estágios (tabelas IV, V e VI). Os valores do $\mathrm{VEO}_{2}$ mostraram reduções significativas apenas entre as médias obtidas no $2^{\circ} \mathrm{e} 3^{\circ}$ estágios (tabelas $\mathrm{V}$ e VI). Os valores do equivalente ventilatório para $\mathrm{CO}_{2}$ mostraram elevações significativas apenas na fase pós-II-VTM no $2^{\circ}$ e $3^{\circ}$ estágio (tabelas Ve VI).

\section{Discussão}

A VTM traz, a curto e longo prazo, alterações nas respostas cardiorrespiratórias durante o exercício e em condições de repouso.

Logo após a VTM, registram-se aumento da área valvar mitral, com consequiente queda do gradiente mitral, da pressão capilar pulmonar e aumento do débito cardíaco independente de efeitos na função ventricular esquerda ${ }^{1-3}$. Em conseqüência, tem se verificado melhora do TF após $\mathrm{VTM}^{2,5,8,9,12}$. No presente estudo, a redução do TF não foi significativa, provavelmente, dado o pequeno número de pacientes avaliados; entretanto, houve tendência não significativa neste sentido.

Na estenose mitral, a circulação pulmonar está alterada, com elevações na pressão de artéria pulmonare até mesmo da resistência pulmonar. Durante o exercício, esses valores apresentam elevações desproporcionais ${ }^{2,5,6,13}$. Após comissurotomia mitral ${ }^{14} \mathrm{e}$ após VTM ${ }^{1,2,3,6}$, há redução precoce da pressão de artéria pulmonar e da pressão capilar pulmonar em repouso e durante o exercício dinâmico. Nas primeiras $24 \mathrm{~h}$ após a VTM, há queda progressiva da resistência pulmonar ${ }^{15}$. Em nossos casos, observaram-se alterações significantes do eixo elétrico do coração, com desvio significativo do eixo para a esquerda, logo após a VTM, permanecendo os valores inalterados durante a evolução. Resultados semelhantes foram relatados por Chen e $\mathrm{col}^{12}$. Apesar da involução, em alguns casos os padrões de sobrecarga ventricular direita não mostraram redução significativa.

Em pacientes com hipertensão venocapilar, o exercício físico pode causar aumento do transudato alveolar em intersticial com dispnéia intolerável responsável pela interrupção do esforço em níveis inferiores à capacidade aeróbia máxima ${ }^{16}$. Em nossos pacientes o critério de interrupção do exercício em todos os casos foi a exaustão física. A duração do exercício tem sido correlacionada à gravidade da estenose mitral ${ }^{17,18}$. Em nossos testes, observaram-se elevações significantes no tempo de endurance em fases precoces e tardias após VTM. Elevações tardias do tempo de endurance têm sido descritas ${ }^{2,19,9}$. Tendências não significativas de elevação do tempo de endurance foram verificadas logo após a VTM ${ }^{8,9}$.

Os desníveis do segmento ST verificados durante o TEE não mostraram diferenças significativas na evolução. Desníveis anormais foram mais freqüentes nas fases pré e pós-I-VTM. Em pacientes com estenose mitral descreveram-se testes ergométricos isquêmicos e angina de peito ${ }^{20}$. No entanto, o uso de digitálicos e diuréticos pelos pacientes 
durante este estudo interferiu na interpretação dos resulta$\operatorname{dos}^{21}$. A alta prevalência de testes com respostas anormais de ST e a baixa prevalência de doença coronária aterosclerótica em mulheres jovens levou a não inclusão da coronariografia no protocolo desta pesquisa ${ }^{22,23}$.

Em indivíduos sadios, durante o exercício dinâmico, há elevação da fração de ejeção e do volume sistólico do ventrículo esquerdo ${ }^{24,25}$. Na estenose mitral, a restrição do enchimento sistólico reduz o volume sistólico durante o exercício e o débito cardíaco é dependente da FC e extração periférica $\mathrm{de}_{2}{ }^{13}$. Em casos graves de estenose mitral, há grandes elevações das catecolaminas plasmáticas durante exercício físico $^{26}$. Em geral, não se tem relatado alterações significativas no pico da FC pós-VTM ${ }^{8,9,27}$, ao contrário do que ocorreu neste estudo. Entretanto, em cargas submáximas de trabalho, houve redução significativa da FC a curto e longo prazo. É possível que a redução da FC em cargas de trabalho submáximo esteja relacionada à redução das catecolaminas plasmáticas e do custo energético do exercício, secundário à maior eficiência mecânica. Entretanto, McKay e $\mathrm{col}^{2}$, em cargas submáximas de elevada intensidade, não encontraram reduções da FC após VTM. Os níveis de pico de PA durante o exercício mantiveram-se estáveis após VTM, conforme descrito por outros autores 9,27 .

Não ocorreram elevações significantes do pico de $\mathrm{VO}_{2}$ logo após a VTM. Apesar da redução documentada da PA esquerda e da pressão venosa capilar, resultados semelhantes foram encontrados por outros autores ${ }^{8,9,12}$. A longo prazo, os valores de pico do $\mathrm{VO}_{2}$ mostraram-se elevados, de acordo com a literatura ${ }^{9,12,27}$. Douard e col $^{27}$ não encontraram correlação entre a área da valva mitral ou gradiente mitral com as alterações do $\mathrm{VO}_{2}$ pico $^{27}$. Marzo e col mostraram correlação significante entre as variações da área valvar mitral e as variações do pico de $\mathrm{VO}_{2}$, três meses após VTM ${ }^{9}$. No presente estudo, os pacientes apresentaram melhora do desempenho cardiovascular, apesar da tendência à reestenose parcial, com elevações tardias dos valores de pico de $\mathrm{VO}_{2}$, VE e $\mathrm{VECO}_{2}$, sem alterações do limiar anaeróbio. A inatividade física prolongada provoca hipotrofia muscular e redução da vascularização e do potencial oxidativo da musculatura esquelética; após condicionamento físico, ocorre hipertrofia muscular, aumento da densidade capilar, do volume das mitocôndrias musculares e da capacidade oxidativa mitocondrial ${ }^{28,29}$. Logo após a VTM, Marzo e $\operatorname{col}^{9}$ não encontraram alterações na oxigenação do músculo vasto lateral durante o exercício; entretanto, três meses após, a desoxigenação em cargas submáximas mos- trou tendência a melhora, sugerindo melhor perfusão tissular em esforços submáximos realizados após VTM. No mesmo estudo, a desoxigenação do músculo serrátio anterior mostrou quedas significativas durante o esforço logo após a VTM. Com base nesses achados, atribuiu-se o alívio dos sintomas a curto prazo, à redução do trabalho respiratório e, a longo prazo, às alterações da musculatura esquelética associadas à redução do trabalho respiratório ${ }^{9}$. É provável que elevações precoces do pico de $\mathrm{VO}_{2}$ não tenham ocorrido por falta de tempo suficiente para a adaptação da musculatura periférica aos padrões de vida compatíveis com a redução abrupta do TF. A longo prazo, com eventuais mudanças nos hábitos de vida e conseqüente aumento do grau de atividade física, teriam ocorrido adaptações periféricas responsáveis pelo aumento tardio da capacidade aeróbia.

Em cargas submáximas de trabalho observaram-se reduções significativas na $\mathrm{FC}, \mathrm{VO}_{2}$ e VE. É provável que a redução do custo metabólico do exercício, com o incremento da eficiência mecânica e com a redução do esforço respiratório esteja implicada na gênese destes eventos. O custo respiratório elevado pré-VTM seria atribuído à redução da complacência pulmonar e da hiperventilação secundárias à hipertensão veno capilar ${ }^{8}$. Em pacientes com estenose mitral a elevação exagerada da VE durante o exercício tem sido atribuída ao aumento do estímulo aos quimiorreceptores ${ }^{30}$, à taquipnéia secundária à redução da complacência pulmonar ${ }^{31} \mathrm{e}$ à congestão pulmonar com estímulo de receptores capilares pulmonares ${ }^{32}$.

Inúmeros fatores neuro-humorais e endócrinos devem estar envolvidos nas adaptações fisiológicas durante o exercício após VTM. Ishikura e $\operatorname{col}^{33}$ detectaram redução dos níveis séricos do peptídeo auricular natriurético durante VTM. Após VTM, os incrementos ocorridos durante o exercício foram menores e guardaram correlação significativa com gradientes de pressão na valva mitral ${ }^{34}$.

As adaptações cardiorrespiratórias precoces e tardias após a VTM por cateter-balão constituem um campo aberto para pesquisas sobre os mecanismos envolvidos na gênese dos sintomas e sinais; o conhecimento exato destes mecanismos deverá trazer maior eficácia ao tratamento clínico e intervencionista da estenose mitral.

\section{Agradecimentos}

Ao Sr. Wilson Roberto Cavalheiro pela análise estatística efetuada.

\section{Referências}

1. Palacios I, Block P, Brandi S et al - Percutaneous balloon valvotomy for patients with severe mitral stenosis. Circulation 1987; 75: 778-84.

2. McKay CR, Kawanishi DT, Kotlewski A et al-Improvement in exercise capacity and exercise hemodynamics 3 months after double-balloon, catheter balloon valvuloplasty treatment of patients with symptomatic mitral stenosis. Circulation 1988; 77: 1013-21.
3. Murat-Tuzcu E, Block P, Griffin B, Newell J, Palacios I - Immediate and long-term outcome of percutaneous valvotomy in patients 65 years and older. Circulation 1992; 85: 963-71.

4. Hung J, Chern M, Wu J et al - Short and long term results of catheter balloon mitral commissurotomy. Am J Cardiol 1991; 67: 854-62.

5. Leavitt JI, Coats MH, Falk RH - Effects of exercise on transmitral gradient and 
pulmonary artery pressure in patients with mitral stenosis or a prosthetic mitral valve. J Am Coll Cardiol 1991; 17: 1520-6.

6. Tamai J, Nagata S, Akaike Met al - Improvement in mitral flow dynamics during exercise after percutaneous transvenous mitral commissurotomy: noninvasive evaluation using continuous wave Doppler technique. Circulation 1990; 81: 46-51.

7. Kasalicky J, Hurych J, Widimsky J et al - Left heart haemodynamics at rest and during exercise in patients with mitral stenosis. Br Heart J 1968; 30: 188-95.

8. Martinez EE, Barros TL, Santos DV et al - Cardiopulmonary exercise testing early after catheter-balloon mitral valvuloplasty in patients with mitral stenosis. Int J Cardiol 1992; 37: 7-13.

9. Marzo KP, Herrmann HC, Mancini DM-Effect of balloon mitral valvuloplasty on exercise capacity, ventilation and skeletal muscle oxygenation. J Am Coll Cardiol 1993; 21: 856-65.

10. Regazzini M - Contribuição ao estudo da evolução de portadores de estenose valvar mitral submetidos à valvoplastia por cateter-balão através de procedimentos não invasivos. (Tese Mestrado) São Paulo: Escola Paulista de Medicina, 1993: 95p.

11. Hatle L, Angelsen B - Doppler Ultrasound in Cardiology, $2^{\text {nd }}$ ed. Philadelphia: Lea \& Febiger, 1985: 118-28.

12. Chen CR, Cheng TO, Chen JY,Zhou YL, Mei J, MaTZ-Long-term results of percutaneous mitral valvuloplasty with the Inoue balloon catheter. Am J Cardiol 1992; 70: 1445-8

13. Gorlin R, Sawyer CG, Haynes FW, Goodale WT, Dexter L-Effects of exercise on circulatory dynamics in mitral stenosis. Am Heart J 1951; 41: 192-9.

14. Feigenbaum H, Linback RE, Nasser WK-Hemodynamic Studies before an after instrumental mitral commissurotomy. Circulation 1968; 38: 261-8.

15. Block PC, Palacios IF - Pulmonary vascular dynamics after percutaneous mitral valvotomy. J Thorac Cardiovasc Surg 1988; 96: 39-46.

16. Gilbert CA, Schilant RC-Role of exercise testing in the preoperative and postoperative assessment of patients with noncoronary disease. Cardiovasc Clin 1978; 9: $173-8$.

17. Almendral JM, Garcia J, Andocin JM, Schanez-Cascos A, DeRabogo P-Treadmill stress testing in the evaluation of patients with valvular heart disease: possible role in the assessment of functional capacity and severity of the lesion. Cardiology 1982; 69: 42-51.

18. Vacek JL, Valentin-Stone P, Wolfe M, Davis WR - The value of standardized exercise testing in the noninvasive evaluation of mitral stenosis. Am J Med Sci 1986 292: 335-43.

19. Stefanades C, Stratos C, Pitsavos C el al - Retrograde nontranseptal balloon mi- tral valvuloplasty: immediate results and long-term follow-up. Circulation 1992; 85: 1760-7.

20. Mittal SR, Mittal B, Singh NP-Exercise-induced myocardial ischemia in young patients of rheumatic mitral stenosis. Int J Cardiol 1995; 50: 198

21. Sundqvist K - Effect of digoxin on the eletrocardiogram at rest and during exercise in health subjects. Am J Cardiol 1986; 57: 661-8.

22. Diamond GA, Forrester JS - Analysis of probabylity as and in the clinical diagnosis of coronary artery disease. N Eng Med J 1979; 300: 1350-9.

23. Morise AP, Duval RD - The estimation of post-test probability of coronary disease following exercise testing using the sequential application of two Bayesian methods. Am Heart J 1990; 120: 1292-7.

24. Sharma B, Goodwin JF, Raphael MJ et al - Left ventricular angiography on exercise. A new method of assessing left ventricular function in ischemic heart disease. Br Heart J 1976; 38: 59-66.

25. Rerych SK, Scholz PM, Newman GE et al - Cardiac function at rest and during exercise in normals and in patients with coronary heart disease: evaluation by radionuclide angiocardiography. Ann Surg 1978; 187: 449-57.

26. Ikeda J, Haneda H, Shirato Ket al - The degree of increment in plasma catecholamine in patients with mitral stenousis by mild exercise. Am Heart J 1987; 113: 1103-9.

27. Douard H, Gilles YM, Choussat A, Broustet JP - Lack of correlation between haemodynamic and cardiopulmonary exercise capacity improvement after catheter-balloon mitral valvuloplasty. Eur Heart J 1995; 16: 1375-9.

28. Andersen P, Henriksson J - Capillary supply of the quadriceps femoris muscle of man: adaptive response to exercise. J Physiol 1977; 270: 677-86.

29. Houston M, Bentzen H, Larsen H - Interrelationship between skeletal muscle adaptations and performance as studied by detraining and retraining. Acta Physiol Scand 1974; 105: 163-71.

30. Donald KW, Gloster J, Harris EA, Reeves J, Harrias P - The production of lactic acid during exercise in normal subjects and in patients with rheumatic heart disease. Am Heart J 1961; 62: 494-519.

31. Palmer WH, Gee JBL, Mills FC, Bates DB - Disturbances of pulmonary function in mitral valve disease. Can Med Assoc J 1963; 89: 744-50.

32. Paintal AS - Mechanism of stimulation F type J pulmonary receptors. J Physiol 1969; 203: 511-32.

33. Ishikura FN, Nagata S, Hirata $Y$ et al - Rapid reduction of plasma atrial natriuretic peptide levels during percutaneous transvenous mitral commissurotomy in patients with mitral stenosis. Circulation 1989; 79: 47-50.

34. Ishikura FN, Nagata S, Tamai J, Miyatake K, Akaike M - Effects of percutaneous transvenous mitral commissurotomy on levels of plasma atrial natriuretic peptide levels during exercise. Am J Cardiol 1989; 67: 74-8. 